\title{
Comparative Study of Field-Oriented Control in Different Coordinate Systems for DTP-PMSM
}

\author{
Ping Zhang ${ }^{*}$, Wei Zhang ${ }^{* *}$, and Xiaofeng Shen ${ }^{* * *}$
}

\begin{abstract}
This paper performs two kinds of Field-Oriented Control (FOC) for dual three phase permanent magnet synchronous motor (DTP-PMSM).The first is based on vector space decomposition to study the effect of current harmonics on electromechanical energy conversion. And the second presents the coupling relations between two sets of windings using two d-q transformation. And then this paper has deeply studied the differences between these two strategies, the different effect on the control of harmonic current and the reason for these differences. MATLAB-based Simulation studies of a 3KW DTP-PMSM are carried out to verify the analysis of differences between the two FOC strategies.
\end{abstract}

Keywords: DTP-PMSM, FOC, Vector space decomposition, Dual dq transformation, Current harmonics, Torque ripple

\section{Introduction}

Compared with three-phase motor drive system, multiphase motor drive has the advantages such as low powerhigh power, high reliability [1-5].And DTP-PMSM has become one of the most popular because it can effectively weaken or even eliminate $5^{\text {th }}$ and $7^{\text {th }}$ motor stator current harmonics. Direct torque control and current vector control are the most common control modes for DTP-PMSM.

For vector control, several strategies such as vector space decomposition (VSD) strategy $[1,6,7]$ and dual synchronous rotating coordinate transformation (DSRCT) strategy $[5,8]$ are proposed during the last year. Vector space decomposition strategy proposed by Yifan Zhao etc has good effect on the control of current harmonic in different subspaces, while DSRCT strategy focus on the internal coupling structure and treat the motor as the organic combination of two three-phase motor that are coupled to each other.

These strategies get good control effect and stable torque. But the different current harmonics distribution and torque ripple under different coordinate system have not been compared.

Therefore this paper will firstly analysis the two common coordinate systems, then study the reasons leading to these differences, and finally give the applicable occasions that the two strategies can be applied to.

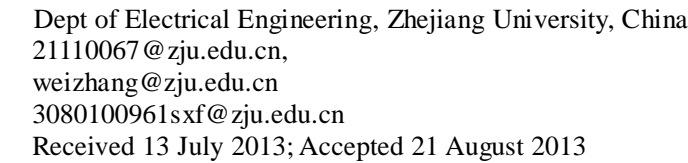

\section{FOC Based On VSD}

The structure of dual three-phase permanent magnet synchronous motor and the drive system are shown in Figure.1. The first set of winding is consist of $A B C$ windings and the other one is made up of DEF windings, which is behind the first set by $30 \mathrm{o}$ angle in the space. In the following analysis, magnetic saturation and iron losses are neglected $[1,5,7]$.

The voltage equations and the flux equations of the DTPPMSM are as follow [4, 9]:

$$
\left\{\begin{array}{l}
{\left[u_{s}\right]=\left[R_{s}\right] \cdot\left[i_{s}\right]+p \cdot\left[\psi_{s}\right]} \\
{\left[\psi_{s}\right]=\left[L_{s s}\right] \cdot\left[i_{s}\right]+\psi_{f} \cdot\left[F\left(\theta_{s}\right)\right]}
\end{array}\right.
$$

Respectively, [us], [is] and [ $\psi \mathrm{s}]$ are the stator voltage,current and flux matrix. [Rs] is the stator resistance matrix and [Lss] is the self-inductance matrix of the stator. $\psi \mathrm{f}$ is the permanent magnet flux of rotor with the coefficient. By vector space decomposition, the DTPPMSM can be decoupled from six-dimension to three mutually two-dimensional orthogonal sub-planes, d-q plane, $\mathrm{z1}-\mathrm{z} 2$ plane and o1-o2 plane. The fundamental variables and the harmonics with order of $\mathrm{k}=12 \mathrm{~m} \pm 1(\mathrm{~m}=1,2,3 \ldots)$ are transformed into the d-q sub-plane, which produce a rotating MMF in the machine air gap and is related to electromechanical energy conversion [2]. And the harmonics with order $6 \mathrm{~m} \pm 1(\mathrm{~m}=1,3,5 \ldots)$ in the $\mathrm{z} 1-\mathrm{z} 2$ plane will not generate any rotating MMF in the air gap and can be seen as a new type of zero-sequence component, while the conventional zero sequence components with 
order of $3 \mathrm{~m}(\mathrm{~m}=1,2,3 \ldots)$ are mapped into the o1-o2 subplane[1,6,7].
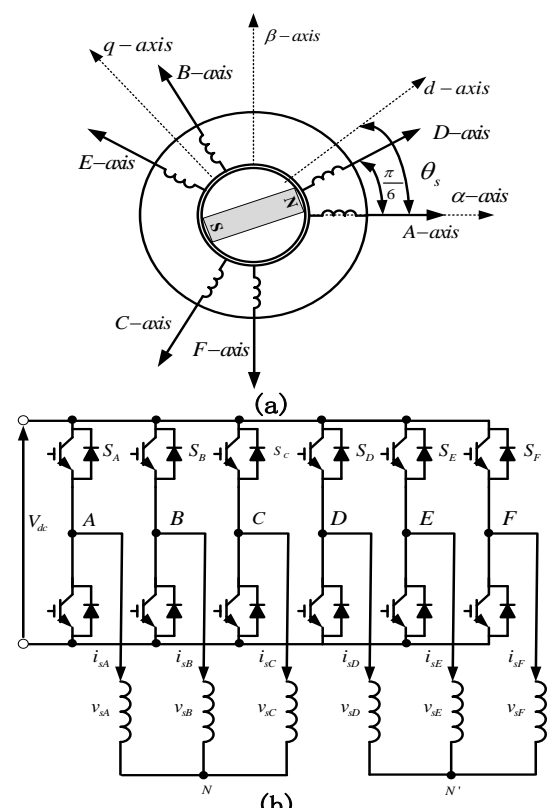

(b)

Fig. 1. Dual Y shift 30 degree PMSM and the drive system (a) winding structure of DTP-PMSM, (b) drive system matrix

And then equation (2) is applied to the model of DTPPMSM to achieve the decoupled motor model in synchronous rotating coordinate system according to the theory of vector space decomposition proposed by Yifan Zhao[1,8].

$$
T_{652 r}=\sqrt{\frac{1}{3}}\left[\begin{array}{cccccc}
\cos \theta_{s} & \cos \left(\theta_{s}-\frac{4 \pi}{6}\right) & \cos \left(\theta_{s}-\frac{8 \pi}{6}\right) & \cos \left(\theta_{s}-\frac{\pi}{6}\right) & \cos \left(\theta_{s}-\frac{5 \pi}{6}\right) & \cos \left(\theta_{s}-\frac{9 \pi}{6}\right) \\
-\sin \theta_{s} & -\sin \left(\theta_{s}-\frac{4 \pi}{6}\right) & -\sin \left(\theta_{s}-\frac{8 \pi}{6}\right) & -\sin \left(\theta_{s}-\frac{\pi}{6}\right) & -\sin \left(\theta_{s}-\frac{5 \pi}{6}\right) & -\sin \left(\theta_{s}-\frac{9 \pi}{6}\right) \\
1 & \cos \frac{8 \pi}{6} & \cos \frac{4 \pi}{6} & \cos \frac{5 \pi}{6} & \cos \frac{\pi}{6} & \cos \frac{9 \pi}{6} \\
0 & \sin \frac{8 \pi}{6} & \sin \frac{4 \pi}{6} & \sin \frac{5 \pi}{6} & \sin \frac{\pi}{6} & \sin \frac{9 \pi}{6} \\
1 & 1 & 1 & 0 & 0 & 0 \\
0 & 0 & 0 & 1 & 1 & 1
\end{array}\right]
$$

Motor model of DTP-PMSM in the $d-q$ sub-plane is shown as the follow $[1,7]$ :

$$
\left\{\begin{array}{l}
u_{s d}=R_{s} \cdot i_{s d}+p \llbracket \psi_{s d}-\omega \cdot \psi_{s q} \\
u_{s q}=R_{s} \cdot i_{s q}+p \sqsubset \psi_{s q}-\omega \cdot \psi_{s d} \\
\psi_{s d}=L_{d} \cdot i_{s d}+\sqrt{3} \cdot \psi_{f} \\
\psi_{s q}=L_{q} \cdot i_{s q}
\end{array}\right.
$$

The voltage equations in the $\mathrm{z} 1-\mathrm{z} 2$ sub-plane:

$$
\left[\begin{array}{l}
u_{s z_{1}} \\
u_{s z_{2}}
\end{array}\right]=\left[\begin{array}{cc}
R_{s}+L_{l s} \cdot p & 0 \\
0 & R_{s}+L_{l s} \cdot p
\end{array}\right] \cdot\left[\begin{array}{l}
i_{s z_{1}} \\
i_{s z_{2}}
\end{array}\right]
$$

The voltage equations in the o1-o2 sub-plane:

$$
\left[\begin{array}{l}
u_{s o_{1}} \\
u_{s o_{2}}
\end{array}\right]=\left[\begin{array}{cc}
R_{s}+L_{l s} \cdot p & 0 \\
0 & R_{s}+L_{l s} \cdot p
\end{array}\right] \cdot\left[\begin{array}{l}
i_{s o_{1}} \\
i_{s o_{2}}
\end{array}\right]
$$

It is can be concluded that the interaction between the stator windings and rotor windings only occurs in the $d-q$ sub-plane. All of the motor component expressed in the equations (4) and (5) will generate just the harmonic current in the stator. And the motor component in the $\mathrm{z} 1-\mathrm{z} 2$ harmonic subspace should be limited as small as possible to reduce the harmonic $[2,4,9]$.

The electromagnetic torque can be achieved by the follow format:

$$
T_{e}=n_{p}\left[\sqrt{3} \psi_{f} \cdot i_{s q}+\left(L_{s d}-L_{s q}\right) i_{s q} \cdot i_{s d}\right]
$$

The control frame of DTP-PMSM based on VSD is shown in the Fig. 2 The reference of d-axis current is set to zero to ensure that the electromagnetic torque is proportional to q-axis according to the equation $6[7,9]$. And voltage component in the $\mathrm{z} 1-\mathrm{z} 2$ subspace is also controlled to zero to weaken the current harmonics that take no part in the electromechanical energy conversion $[1,10]$.

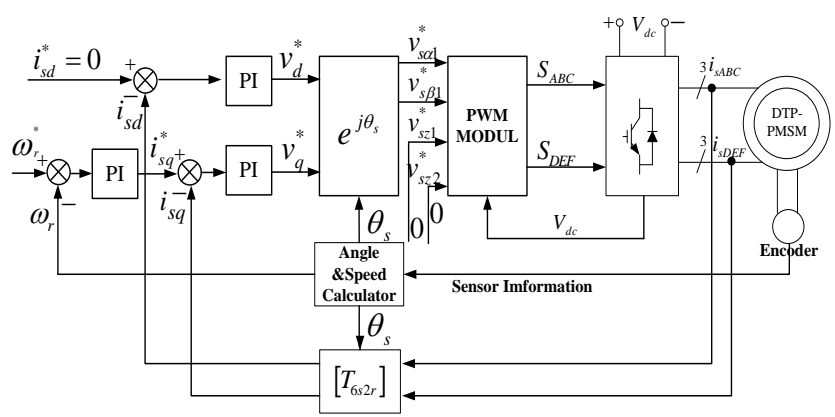

Fig. 2. Control frame of DTP-PMSM based on VSD

\section{FOC Based On DSRCT}

In fact, the two sets of three-phase windings of DTPPMSM are symmetrical and their neutral are also independent to each other. So the DTP-PMSM can be seen as two separate systems with decoupling between the two sets of windings [5]. This means that the motor can be controlled in two separate $\mathrm{d}-\mathrm{q}$ reference frames simultaneously. The conventional rotating coordinate transformation is easily applied to each set of DTP-PMSM. 
(7) is the coordinate transformation format for the first set of windings and (8) is for the other one. The Fig. 3 is schematic diagram of dual $\mathrm{d}-\mathrm{q}$ coordinate system for dual three-phase permanent magnet synchronous motor. $\mathrm{S}$ is the angle between the winding of $A$ phase and the $\mathrm{d} 1$-axis.And the $\mathrm{d} 1 \mathrm{-q} 1$ rotating coordinate system for $\mathrm{ABC}$ windings is 30 degrees ahead of the $\mathrm{d} 2-\mathrm{q} 2$ rotating coordinate system for DEF windings[1,5,10]. When the transformation is applied to both winding sets, the stator voltage equations in $\mathrm{d}-\mathrm{q}$ reference frame can be derived by:

$$
T_{3 s 2 r}^{1}=\sqrt{\frac{2}{3}}\left[\begin{array}{ccc}
\cos \theta_{s} & \cos \left(\theta_{s}-\frac{2}{3} \pi\right) & \cos \left(\theta_{s}+\frac{2}{3} \pi\right) \\
-\sin \theta_{s} & -\sin \left(\theta_{s}-\frac{2}{3} \pi\right) & -\sin \left(\theta_{s}+\frac{2}{3} \pi\right) \\
\frac{1}{\sqrt{2}} & \frac{1}{\sqrt{2}} & \frac{1}{\sqrt{2}}
\end{array}\right]
$$

$$
T_{3 s 2 r}^{2}=T_{3 s 2 r}^{1}\left(\theta_{s}-\pi / 6\right)
$$

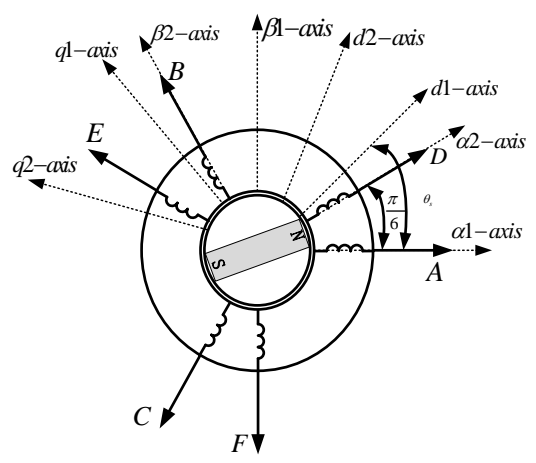

Fig. 3. Schematic diagram of dual dq transformation of DTP-PMSM

$$
\left\{\begin{aligned}
u_{s d 1} & =R_{s} i_{s d 1}+p \llbracket \psi_{s d 1}-\omega \psi_{s q 1} \\
& =R_{s} i_{s d 1}+L_{d}\left(p \cdot i_{s d 1}\right)+M_{d}\left(p \cdot i_{s d 2}\right)-\omega L_{q} i_{q 1}-\omega M_{q} i_{q 2} \\
u_{s q 1} & =R_{s} i_{s q 1}+p \llbracket \psi_{s q 1}+\omega \psi_{s d 1} \\
& =R_{s} i_{s q 1}+L_{d}\left(p \cdot i_{s q 1}\right)+M_{q}\left(p \cdot i_{s q 2}\right)+\omega L_{d} i_{d 1}+\omega M_{d} i_{d 2 f}+\sqrt{\frac{3}{2}} \varphi_{f} \\
u_{s d 2} & =R_{s} i_{s d 2}+p \llbracket \psi_{s d 2}-\omega \psi_{s q 2} \\
& =R_{s} i_{s d 2}+L_{d}\left(p \cdot i_{s d 2}\right)+M_{d}\left(p \cdot i_{s d 1}\right)-\omega L_{q} i_{q 2}-\omega M_{q} i_{q 1}+\sqrt{\frac{3}{8}} \varphi_{f} \\
u_{s q 2} & =R_{s} i_{s q 2}+p \llbracket \psi_{s q 2}+\omega \psi_{s d 2} \\
& =R_{s} i_{s q 2}+L_{d}\left(p \cdot i_{s q 2}\right)+M_{d}\left(p \cdot i_{s q 1}\right)+\omega L_{d} i_{d 2}+\omega M_{d} i_{d 1}+\sqrt{\frac{9}{8}} \varphi_{f}
\end{aligned}\right.
$$

Where $\omega$ is the electrical angular speed of the rotor, $\psi$ is the flux linkage, $\mathrm{L}$ is the self-inductance, $\mathrm{M}$ is the mutual inductance, $i$ is the current, the subscript $d 1$ and $q 1$ refers to the $\mathrm{d}$-axis and q-axis component of the first winding set respectively and $\mathrm{d} 2$ and $\mathrm{q} 2$ to the $\mathrm{d}$-axis and $\mathrm{q}$-axis component of the second winding set.

Some conclusions about the electromagnetic coupling of the winding sets of the DTP-PMSM can be easily got from (9).The DTP-PMSM is a multi-input multi-output and fourorder nonlinear system. And then this paper derives the voltage compensation terms in (10) to eliminate the couplings as positive feedbacks to the output of the current PI-regulators.

$$
\left\{\begin{array}{l}
u_{s d 1}^{d e c}=M_{d} \cdot\left(p \cdot i_{s d 2}\right)-\omega L_{q} i_{s q 1}-\omega M_{q} i_{s q 2} \\
u_{s q 1}^{d e c}=M_{q} \cdot\left(p \cdot i_{s q 2}\right)+\omega L_{d} i_{s d 1}+\omega M_{d} i_{s d 2}+\sqrt{\frac{3}{2}} \varphi_{f} \\
u_{s d 2}^{d e c}=M_{d} \cdot\left(p \cdot i_{s d 1}\right)-\omega L_{q} i_{s q 2}-\omega M_{q} i_{s q 1}+\sqrt{\frac{3}{8}} \varphi_{f} \\
u_{s q 2}^{d e c}=M_{q} \cdot\left(p \cdot i_{s q 1}\right)+\omega L_{d} i_{s d 2}+\omega M_{d} i_{s d 1}+\sqrt{\frac{9}{8}} \varphi_{f}
\end{array}\right.
$$

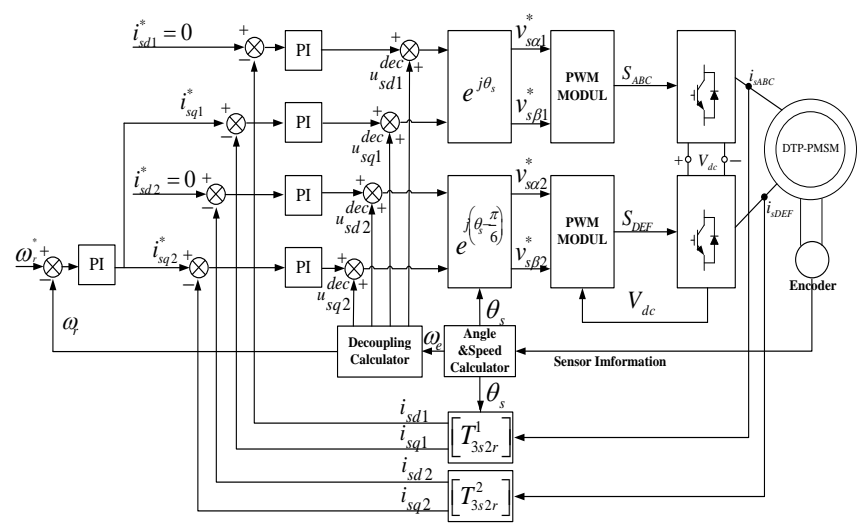

Fig. 4. Control frame of DTP-PMSM based on DSRCT

Fig. 4 is the control frame of DTP-PMSM based on current compensation by winding decoupling. In this strategy, the conventional three-phase motor coordinate transformation is applied to each set of three-phase symmetrical windings to get two two-dimensional and mutually coupled d-q subspaces. And the decoupling calculator is used to calculate the coupling component to compensate the reference voltage. The output of the speed controller is the reference value of the q1-axis and q2-axis current. And four current variables have been required to meet the constraints derived in (11):

$$
\left\{\begin{array}{l}
i_{s d 1}=i_{s d 2}=0 \\
i_{s q 1}=i_{s q 2}
\end{array}\right.
$$

And then the electromagnetic torque is showed as the follow:

$$
T_{e}=n_{p} \psi_{f} i_{s q 1}(\sqrt{3 / 2}+\sqrt{3 / 8}+\sqrt{9 / 8})
$$

Under the terms of the constant permanent magnet flux 
$\psi_{f}$ and pole pairs $n_{p}$ and the constraints derived in (11), the electromagnetic torque is proportional to the q1-axis (q2axis) component of stator current and the instantaneous linear control of the electromagnetic torque can be easily realized with the control of q1-axis stator current.

\section{Comparison of Two FOC}

For the VSD strategy, the fundamental electromagnetic components have been mapped to the $\mathrm{d}-\mathrm{q}$ and they will generate the rotating MMF and complete electromechanical energy conversion $[1,6]$. And the electromagnetic components in other two sub-planes are orthogonal to the d-q sub-plane, therefore will not generate any rotation MMF and provide electromechanical energy [1, 2, 6, 9 ]. But they will generate harmonic currents in the stator windings and increase the stator losses. So the components in the z1-z2 sub-plane should be controlled to zero.

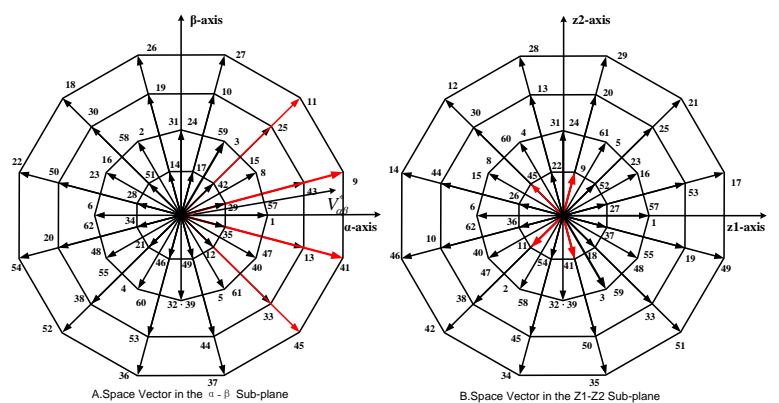

Fig. 5. Schematic diagram of space vectors based on VSD.

(a)Space Vector $s$ in $\alpha-\beta$ Sub-plane.

(b) Space Vectors in Z1-Z2 Sub-plane

In the VSD strategy, four reference vectors, which are adjacent to reference vector $v_{\alpha \beta}{ }^{*}$ and have the maximum magnitude, has been selected in order to achieve maximum utilization of the DC bus voltage in the $\alpha-\beta$ sub-plane show in the Fig. $5[1,2,6]$. In fact, the voltage vectors with maximum amplitude in the $\alpha-\beta$ sub-plane precisely have the minimum magnitude in the $\mathrm{z} 1-\mathrm{z} 2$ plane sub-plane, which ensures the least current harmonic content in the $\mathrm{z} 1-\mathrm{z} 2$ subplane.

When the reference voltage falls in the S1 zone and the angle from $\alpha$-axis to the reference voltage is 10 degrees. The vectors with the number of 45,41,9 and 11 have been selected and working order is 45->41->9->11.

With the same reference voltage of VSD strategy, the action vectors of the CBA windings are 001,011,000 in sequence and the act ion vectors of the FED windings are $101,001,000$ in sequence. The working time and sequence of the vectors are displayed in the Fig. 6.

\begin{tabular}{l|c|c|c|} 
& \multicolumn{4}{|c|}{ Tpwm } \\
\cline { 2 - 4 } CBA Windings & \multicolumn{2}{|c|}{011} & 000 \\
\cline { 2 - 4 } FED Windings & 001 & 001 & 000 \\
\cline { 2 - 4 } & 101 & \multicolumn{2}{|c|}{001}
\end{tabular}

Fig. 6. Vector working sequence diagram for DSRCT

And the vectors mapped into the $\alpha-\beta$ sub-plane are $45-$ $>41->9->3$ in sequence. The vectors with the numb-er 45 , 41 and 9 are the vectors with maximum amplitude and the vector with number 3 has the second minimum amplitude, which is different from the VSD strategy and will lead to different control effect.
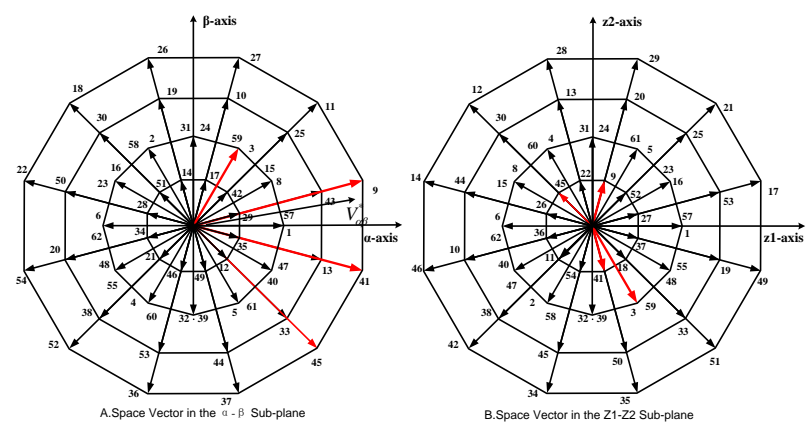

Fig. 7. Schematic diagram of the space vectors on DSRCT (a)Space Vectors of DRSCT strategy in $\alpha-\beta$ Sub-plane. (b)Space Vectors of DRSCT strategy in Z1-Z2 Sub-plane

According to the principles of coordinate transformation for the two Field-Oriented Control strategies, the relationship between two control strategies can be derived from (1), (2), (7) and (8) as follow:

$$
\left\{\begin{array}{l}
i_{d}=\frac{1}{\sqrt{2}}\left(i_{d 1}+i_{d 2}\right) \\
i_{q}=\frac{1}{\sqrt{2}}\left(i_{q 1}+i_{q 2}\right) \\
i_{s 21}=\frac{1}{\sqrt{2}}\left(i_{d 1}-i_{d 2}\right) \cos \theta_{s}-\frac{1}{\sqrt{2}}\left(i_{q 1}-i_{q 2}\right) \sin \theta_{s} \\
i_{s z 2}=-\frac{1}{\sqrt{2}}\left(i_{d 1}-i_{d 2}\right) \sin \theta_{s}-\frac{1}{\sqrt{2}}\left(i_{q 1}-i_{q 2}\right) \cos \theta_{s}
\end{array}\right.
$$

Where the subscript $\mathrm{d}, \mathrm{q}, \mathrm{z} 1$ ad $\mathrm{z} 2$ refers to the components in the vector space decomposition strategy and $\mathrm{d} 1, \mathrm{q} 1, \mathrm{~d} 2$ and $\mathrm{q} 2$ to the components in the dual synchronous rotating coordinate transformation strategy. Taking into account the constraints derived in (11), the current component in the harmonics subspace have been directly controlled to zero, which can ensure less stator loss. And the VSD strategy has just tried to weaken the current harmonics by selecting vectors, whose effect on weakening harmonic is limited.

\section{Simulation Results}


In order to verify the validation of the proposed control strategy, a MATLAB-based simulation of a $3-k W$ s dual three-phase permanent magnet synchronous motor is carried out. The structure is as Fig.1. Vector control based on space vector decomposition strategy and dual synchronous rotating coordinate transformation strategy is applied to the simulation study with the same motor parameters and the same motor speed regulator. The permanent magnet flux is $0.175 \mathrm{~Wb}$; the stator resistance is $1.0 \Omega$; the d-axis and q-axis inductance is $8.5 \mathrm{mH}$, and the leakage inductance is $0.6 \mathrm{mH}$.The motor has four pairs of poles with the moment of inertia of $0.089 \mathrm{~kg}$.m. And the inverter switching frequency is set to $5 \mathrm{k} \mathrm{Hz}$. The given speed is set to $300 \mathrm{rpm}$ with load of $17.5 \mathrm{~N}$.m.

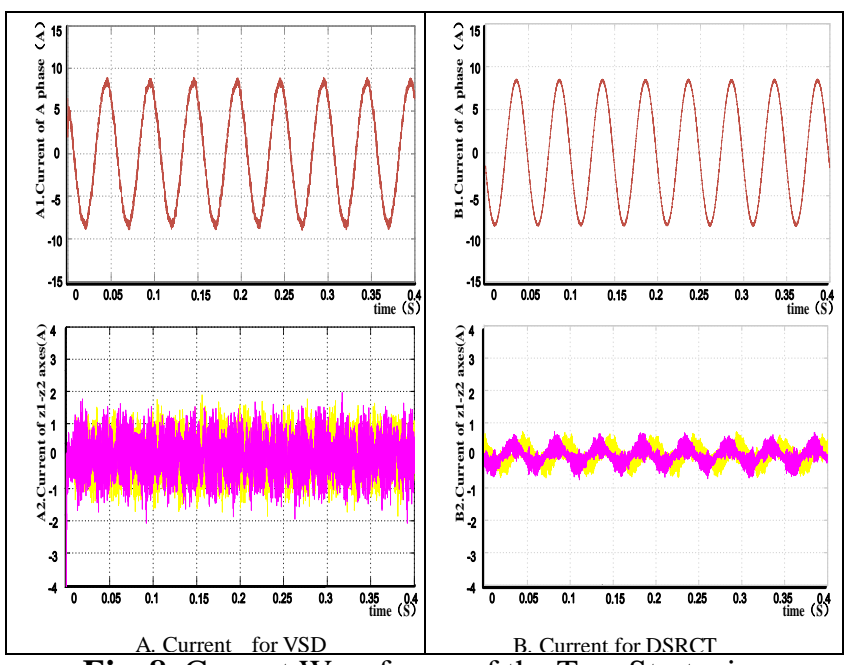

Fig. 8. Current Waveforms of the Two Strategies

(A1), (B1). Current of A phase,

(A2), (B2). Current of z1-z2 subspace

Fig. 8 is the current waveforms of the two field oriented control strategies. And the upper is the current of a phase and the other is the harmonic currents in $\mathrm{z} 1-\mathrm{z} 2$ subspace. The phase current for two strategies are the same with amplitude of 8.4A. The harmonic currents in the harmonic subspace under DSRCT strategy have a amplitude of 0.7A, which is much smaller than the VSD strategy with an amplitude of 1.6A. And this leads to that the phase current in DSRCT strategy is much smoother.

Compared harmonic analysis of the two strategies is showed in the Fig. 9. The total THD of VSD strategy is $4.47 \%$ that is much more than $1.09 \%$ for DSRCT strategy. Some conclusions about current harmonics' distribution can be immediately made from Fig. 9. The current harmonics for VSD strategy are distributed in the order of $6 \mathrm{~m} \pm 1(\mathrm{~m}=1$, $3,5 \ldots)$ that is just in the $\mathrm{z} 1-\mathrm{z} 2$ plane. Furthermore, the high-order and the low-order harmonics are all involved, while the most harmonics of DSRCT strategy is distributed in low order of $5^{\text {th }}, 7^{\text {th }}, 11^{\text {th }}$ and etc. This is because that DSRCT strategy has directly controlled the current harmonics according to (13). Consequently, the DSRCT strategy has achieved less motor loss.
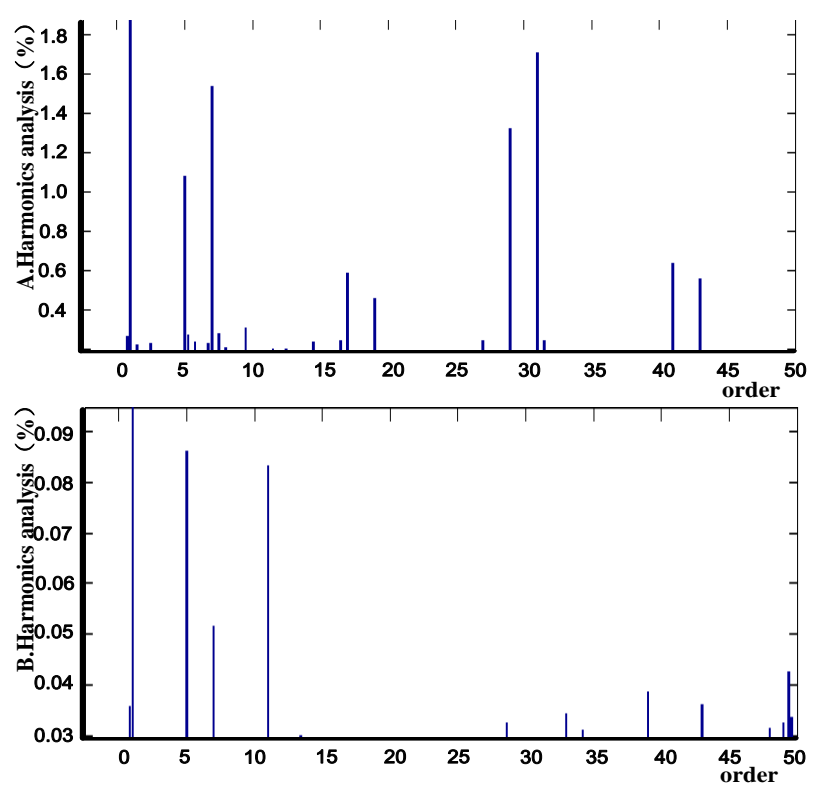

Fig. 9. Harmonic Analysis of the Two Strategies

(A). Harmonic analysis for VSD strategy

(B). Harmonic analysis for DSRCT strategy

Fig. 10 shows the steady-state torque pulsation for the two control strategies. The steady-state torque ripple under the DSRCT strategy is 0.4 N.m compared with 0.6 N.m torque pulsation for the VSD strategy.
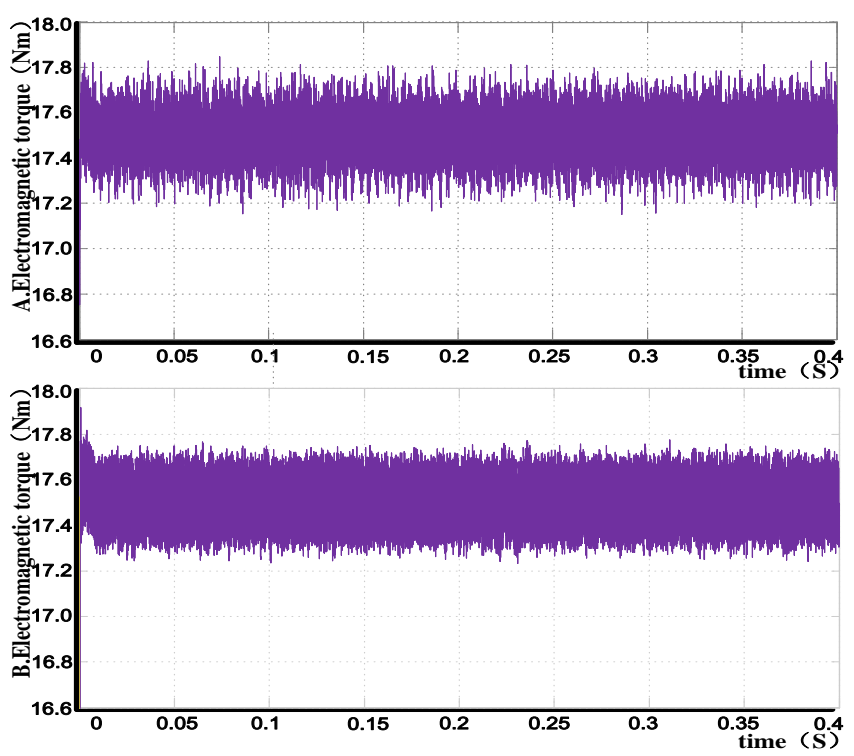

Fig. 10. Steady-state electromagnetic torque ripple

(A).Torque ripple for VSD strategy. (B).Torque ripple for DSRCT strategy 


\section{Conclusion}

This paper has used the vector space decomposition and dual synchronous rotating coordinate transformation to discuss the Field-Oriented control for dual-phase permanent magnet synchronous motor and achieve instantaneous linear control of the electromagnetic torque. And the relationship and the differences between the two field oriented control strategies have been deeply studied. MATLAB-based simulation is carried out to verify the differences between the two strategies. With direct control on the current harmonic in the $\mathrm{z} 1-\mathrm{z} 2$ subspace, DSRCT strategy has achieved better effects on weakening harmonic and can be easily extended to any other multiphase motor with the phase number of multiples of three. In fact, the more accurately and effectively the current variables in $\mathrm{z} 1$ $\mathrm{z} 2$ plane is controlled to zero, the less the harmonic with order of $6 \mathrm{~m} \pm 1$ will be for VSD strategy. And more study can be focused on the control of variables in $\mathrm{z} 1-\mathrm{z} 2$ plane to weaken the harmonic.

\section{References}

[1] Yifan Zhao, Thomas A. Lipo. Vector PWM Control of Dual Three Phase Induction Machine Using Vector Space Decomposition [J]. IEEE Trans. Ind. Appl.1995, 31(5):11001109.

[2] E.A. Klingshirn.High Phase Order Induction Motors Part IDescription and Theoretical Considerations [J].IEEE Trans. Power Apparatus and Systems, 1983,102(1): 47-53.

[3] R. Bojoi, F.Profumo, A. Tenconi.Digital Synchronous Frame Current Regulation for Dual Three-phase Induction Motor Drives. Elettrica IndustrialeC.so Duca degli Abruzzi, 24,10129, Torino, Italy.

[4] E. Levi, "Multiphase electrical machines for variable-speed applications," IEEE Trans. Ind. Appl., vol. 55, pp. 1893-1909, May 2008.

[5] J. Karttunen, S. Kallio, P. Peltoniemi. Dual Three-Phase Permanent Magnet Synchronous Machine Supplied by Two independent Voltage Source Inverters.Appeenranta University of Technology, IEEE Trans. International Symposium on Power Electronics, 2012,p741-747.

[6] M. Kamari, M. Keramatzadeh, and R. Kianinezhad, "Space vector double frame field oriented control of six phase induction motors," WSEAS Trans. Syst. Control, vol. 4, no. 3 , pp. 129-139, Mar. 2009.

[7] T.J.E. Miller and M.I. McGilp, "Analysis of multi-phase permanent- magnet synchronous machines," in Int. Conf. Electrical Machines and Systems (ICEMS), 2009, pp. 1-6.

[8] G. Singh, K. Nam, and S. Lim, "A simple indirect fieldoriented control scheme for multiphase induction machine," IEEE Trans. Ind. Electron, vol. 52, pp 1177-1184, Aug. 2005.

[9] R. Bojoi. M. LarZari, F. Rofumo and A. Tenconi, "Digital field oriented control for dual-three Phase induction motor driver, Conf,Rec. IEEEIAS, 2002,Vol.2,pp818-825.
[10] R. Bojoi, EProfumo, A.Tenconi Politecnico di Torino. Digital Synchronous Frame Current Regulation for Dual Three-phase Induction Motor Drives. . 24,10129, Torino, Italy.

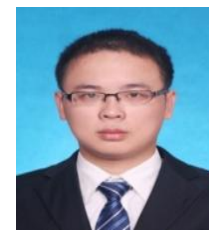

systems.
Ping Zhang received B.S degree in electrical engineering from Shanghai Jiaotong University in 2011. His research interests are emerging energy systems and electric machines and multi-phase motor

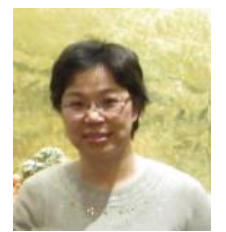

Wei Zhang received B.S degree, M.S. degree and Ph.D. degree in electrical engineering from Zhejiang University. Her fields of interest are power-electronic conversion, high-performance speed servo drives, integrated electronic design, and applications of power quality issues.

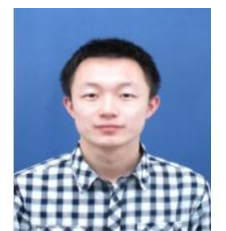

Xiaofeng Shen received B.S degree in electrical engineering from Zhejiang University in 2012. His research interests are emerging energy systems and electric machines. 